\title{
THE SELBERG ZETA FUNCTION AND SCATTERING POLES FOR KLEINIAN GROUPS
}

\author{
PETER A. PERRY
}

In this note we present a polynomial bound on the distribution of poles of the scattering operator for the Laplacian on certain hyperbolic manifolds $M^{n}$ of infinite volume. The motivation is to understand more fully the geometry of the poles of the scattering operator. The proof uses the relationship between poles of the scattering operator and zeros of the Selberg zeta function for geodesic flow on $M^{n}$.

Recall that the classical Selberg zeta function $Z(s)$ [30] is a meromorphic function which describes the lengths $\ell(\gamma)$ of closed geodesics $\gamma$ on a compact surface $S$ :

$$
Z(s)=\prod_{\gamma} \prod_{m=1}^{\infty}\{1-\exp (-(s+m) \ell(\gamma))\}
$$

where the product over $\gamma$ runs over primitive closed geodesics. Crude estimates on the distribution of lengths show that $Z(s)$ is analytic for $\mathfrak{R}(s)>1$; an application of Selberg's trace formula shows that $Z(s)$ extends to a meromorphic function on $\mathbf{C}$ with trivial zeros at the integers $1,0,-1, \ldots$ together with spectral zeros at the numbers $s_{k}$ where $s_{k}\left(1-s_{k}\right)$ is an eigenvalue of the Laplacian on $S[11,20,30]$.

Here we will be concerned with the Selberg zeta functions, introduced by Patterson [25], for certain noncompact hyperbolic manifolds $M^{n}$ of infinite hyperbolic volume. These zeta functions are defined by an infinite product similar to (1) (identical when $n=2$ ); they share important features with the classical zeta function. In particular, their analytic structure is closely tied to the spectrum of the Laplacian on $M^{n}$. However, the Laplacian

Received by the editors December 19, 1989 and, in revised form, November 12, 1990.

1980 Mathematics Subject Classification (1985 Revision). Primary 58G25; Secondary 35P25, 58F20.

Supported in part by NSF Grant DMS-8802668. 
on $M^{n}$ has at most finitely many discrete eigenvalues and absolutely continuous spectrum of infinite multiplicity [13, 14, 15]; thus the dominant contribution to the spectrum is described by the scattering operator $S(s)$ associated to the absolutely continuous spectrum. $S(s)$ is an operator-valued meromorphic function that relates the asymptotic behavior of incoming and outgoing generalized eigenfunctions of the Laplacian (see $[1,16,17,18,19,26$, $28]$ ). The poles of $S(s)$ play a role similar to the role played by eigenvalues of the Laplacian for a compact surface; the zeta function has spectral zeros at poles of the scattering operator together with an infinite sequence of trivial zeros or poles which appear to carry topological information $[25,27,29]$.

In contrast to the eigenvalues of the Laplacian, the poles of the scattering operator are eigenvalues of a nonselfadjoint eigenvalue problem, so that very little is known about their nature and distribution. Recent studies of the scattering operator for acoustical scattering have derived polynomial bounds on the distribution function

$$
\begin{aligned}
N(r)=\#\{k \in \mathbf{C}:|k| \leq & r \text { and } k \text { is a pole } \\
& \text { of the scattering operator }\}
\end{aligned}
$$

(see Melrose [21, 22]), Zworski [33, 34, 35] derives exact leading asymptotics for $N(r)$ in the case of Schrödinger scattering by a compactly supported potential.

Here we prove a polynomial bound for the distribution of poles of the scattering operator $S(s)$. We know of no previous result of this type for the Laplacian on a hyperbolic manifold. Using this bound, one should be able to prove a trace formula involving the poles of the scattering operator and obtain finer information on the distribution of poles (cf. [21, 22, 3] for trace formulas in acoustical scattering and $[10,12]$ for their application to analysis of poles of the scattering operator in certain acoustical problems).

To state our result more precisely, we first recall some notions of hyperbolic geometry. Model hyperbolic space $\mathbf{H}^{n}$ as the unit ball $\mathbf{B}^{n} \subset \mathbf{R}^{n}$ with the Poincare metric

$$
d s^{2}=4|d x|^{2} /\left(1-|x|^{2}\right)^{2},
$$

where $|d x|^{2}$ is the Euclidean metric on $\mathbf{R}^{n}$. Then $S^{n-1} \subset \mathbf{R}^{n}$ is the boundary at infinity for $\mathbf{H}^{n}$, and the isometries of $\mathbf{H}^{n}$ are 
Möbius transformations leaving $\mathbf{B}^{n}$ invariant. A discrete group $\Gamma$ of isometries of $\mathbf{H}^{n}$ is called geometrically finite if it admits a fundamental domain bounded by finitely many geodesic hyperplanes. The limit set $\Lambda_{\Gamma}$ is the set of accumulation points in $S^{n-1}$ of $\Gamma$ orbits of points in $\mathbf{H}^{n}$; it is a closed, $\Gamma$-invariant set. The convex hull of the limit set, $C H\left(\Lambda_{\Gamma}\right)$, is the smallest hyperbolically convex subset of $\mathbf{H}^{n}$ whose boundary (viewed as a subset of $\mathbf{R}^{n}$ ) contains $\Lambda_{\Gamma} \cdot C H\left(\Lambda_{\Gamma}\right)$ is $\Gamma$-invariant; the quotient $F N=C H\left(\Lambda_{\Gamma}\right) / \Gamma$ is called the Fenchel-Nielsen region. Closed geodesics on $M^{n}$ lift to geodesics in $\mathbf{H}^{n}$ with both endpoints in the limit set; such geodesics are contained in $C H\left(\Lambda_{\Gamma}\right)$, so that closed geodesics of $M^{n}$ lie in $F N$. A geometrically finite discrete group $\Gamma$ is called convex co-compact if $F N$ is a compact subset of $\mathbf{H}^{n} / \Gamma$.

We consider quotients $M^{n}=H^{n} / \Gamma$ where

$\Gamma$ is geometrically finite, convex co-compact, and torsion-free, and $\operatorname{vol}\left(\mathbf{H}^{n} / \Gamma\right)=\infty$.

The no-torsion condition guarantees that $M^{n}$ is a Riemannian manifold. The convex co-compactness implies that $M^{n}$ has no cusps. Since $F N$ is compact, the closed geodesics of $M^{n}$ lie in a compact set. Since closed geodesics are dense in the nonwandering set by Anosov's closing lemma [2], the nonwandering set for geodesic flow on the unit tangent bundle of $M^{n}$ is also compact.

The nontrivial geometry of $M^{n}$, as well as its nontrivial dynamics, is concentrated in $F N$. Let $F N_{1}$ be the set of points at distance $\leq 1$ from $F N$. Then $F N_{1}$ is a $C^{1}$ submanifold with boundary and $M^{n}-F N_{1}$ is the union of finitely many connected components diffeomorphic to cylinders $(0,1) \times N_{i}$ where the $N_{i}$ are compact manifolds. The geometric boundary of $M$ is $\partial_{\infty} M^{n}=\bigcup_{i} N_{i}$ and can also be realized as $\Omega_{\Gamma} / \Gamma$ where $\Omega_{\Gamma}=$ $S^{n-1}-\Lambda_{\Gamma}$ is the domain of discontinuity of $\Gamma$ acting in $S^{n-1}$.

The Laplacian on $M^{n}$ has at most finitely many eigenvalues in $\left[0,(n-1)^{2} / 4\right)$ (owing to the infinite volume of $M^{n}[13]$ ), and purely absolutely continuous spectrum on $\left[(n-1)^{2} / 4, \infty\right)$ [14]. The associated scattering operator $S(s)$ is a meromorphic pseudodifferential operator-valued function acting on line bundles over $\partial_{\infty} M^{n}$ (see Agmon [1], Froese, Hislop, and Perry [7], Mandouvalos $[16,17,18,19]$, and Perry $[26,27,28]$ for further discussion). The relation between the scattering operator $S(s)$ and the zeta 
function $Z(s)$ is

Theorem $0.1([25,27])$. Let $M^{n}$ satisfy $(*)$. Then for $\mathfrak{R}(s)=$ $(n-1) / 2, s \neq(n-1) / 2$, the identity

holds.

$$
\frac{Z^{\prime}(s)}{Z(s)}+\frac{Z^{\prime}(n-1-s)}{Z(n-1-s)}=\operatorname{Tr}\left(S(s)^{-1} S^{\prime}(s)\right)
$$

Here the prime denotes differentiation with respect to $s$, and $\operatorname{Tr}$ denotes a suitably renormalized trace (see [27]). In [27] it is shown that the right-hand side has a meromorphic continuation to $\mathbf{C}$ and that each of the nontrivial poles of the scattering operator is a pole of the right-hand side with positive integral residue equal to the dimension of the zero eigenspace of a Fredholm operator that determines the poles of $S(s)$.

On the other hand, the dynamical methods of Ruelle [31] and Fried [8] show that

Theorem 0.2. Let $M^{n}$ satisfy (*). Then the zeta function $Z(s)$ for $M^{n}$ is the quotient of entire functions of order $\leq n$.

Although [8] and [31] analyze zeta functions for geodesic flow on compact manifolds rather than noncompact manifolds, their results remain true for Axiom A flows on noncompact manifolds with analytic stable and unstable foliations and a compact nonwandering set. It is relatively easy to check that these assumptions are satisfied for geodesic flow on the unit tangent bundle of the manifolds $M^{n}$ considered here.

Together with a trivial estimate on the distribution of zeros of entire functions of order $n$, Theorems 0.1 and 0.2 imply:

Theorem 0.3. Let $N(r)$ be the counting function (2) for poles of $S(s)$, i.e.,

$$
N(r)=\#\{s \in \mathbf{C}:|s| \leq r \text { and } s \text { is a pole of } S(s)\} .
$$

Then for any $\epsilon>0$ there is a $C_{\epsilon}>0$ such that

$$
N(r) \leq C_{\epsilon}(1+r)^{n+\epsilon} \text {. }
$$

This result is almost optimal, as the following examples due to Charles Epstein [6] show. Let $\Gamma_{n-1}$ be a discrete group of isometries of $\mathbf{H}^{n-1}$ so chosen that $M^{n-1}=\mathbf{H}^{n-1} / \Gamma_{n-1}$ is a compact Riemannian manifold. There is a natural embedding of $\operatorname{Isom}\left(\mathbf{H}^{n-1}\right)$ in Isom $\left(\mathbf{H}^{n}\right)$ such that, if $\Gamma_{n}$ is the image of $\Gamma_{n-1}$ under this embedding, $\mathbf{H}^{n} / \Gamma_{n}$ is isometric to $\mathbf{R} \times M^{n-1}$ with hyperbolic metric 
$d s^{2}=d \tau^{2}+\cosh ^{2}(\tau) d \omega^{2}$, where $d \omega^{2}$ is the hyperbolic metric on $M^{n-1}$. The zeta function $Z_{\Gamma_{n}}(s)$ is given in terms of the zeta function $Z_{\Gamma_{n-1}}(s)$ by the formula

$$
Z_{\Gamma_{n}}(s)=\prod_{m=0}^{\infty} Z_{\Gamma_{n-1}}(s+m) .
$$

$Z_{\Gamma_{n-1}}(s)$ has spectral zeros due to eigenvalues of the Laplacian on $M^{n-1}$ and topological zeros or poles depending on the dimension and topology of $M^{n-1}$ (see [5, 9, 11, 30]). The spectral zeros of $Z_{\Gamma_{n-1}}(s)$ are those $s_{k}$ on the line $\mathfrak{R}(s)=(n-2) / 2$ for which $s_{k}\left(n-2-s_{k}\right)$ is an eigenvalue of the Laplacian on $M^{n-1}$. Let $s_{k}=(n-2) / 2+i t_{k}$. The $t_{k}$ obey the estimate (Weyl's law for $\left.M^{n-1}[4]\right)$

$$
N\left(t_{k}:\left|t_{k}\right| \leq r\right) \sim r^{n-1} \text {. }
$$

Since the product (3) has zeros $s_{k, m}=-m+s_{k}$ for $m$ a nonnegative integer, it follows that the counting function for these spectral zeros of the product (3) obeys $N(r) \sim r^{n}$. It can be shown by explicit calculation, using the explicit form of the metric to separate variables, that all of these zeros occur as poles of the scattering operator for $M^{n}$.

Details of the proof and the examples described above will appear in [29].

\section{ACKNOWLEDGMENTS}

I am grateful to S. J. Patterson for pointing out the relevance of Fried's work to this problem and to David Fried and S. J. Patterson for helpful discussions.

\section{REFERENCES}

1. S. Agmon, On the spectral theory of the Laplacian on non-compact hyperbolic manifolds, Journées "Équations aux derivées partielles" (Saint Jean de Monts, 1987), Exposée No. XVII, École Polytechnique, Palaiseau, 1987.

2. D. Anosov, Geodesic flows on closed Riemann manifolds with negative curvature, Proc. Steklov Math. Inst. 90 (1967), 1-235.

3. C. Bardos, J. C. Guillot and J. Ralston, La relation de Poisson pour l'équation des ondes dans un ouvert non borné. Application à la théorie de la diffusion, Comm. Partial Differential Equations 7 (1982), 905-958.

4. I. Chavel, Eigenvalues in Riemannian geometry, Academic Press, New York, 1984. 
5. J. Elstrodt, Die Selbergsche Spurformel für kompakte Riemannische Flächen, Jber. d. Dt. Math.-Verein 83 (1981), 45-77.

6. C. Epstein, private communication.

7. R. Froese, P. Hislop, and P. Perry, The Laplace operator on hyperbolic three-manifolds with cusps of non-maximal rank, Invent. Math. (submitted).

8. D. Fried, The Zeta functions of Ruelle and Selberg. I, Ann. Sci. École Norm. Sup. 19 (1986), 491-517.

9. R. Gangolli, Zeta functions of Selberg's type for compact space forms of symmetric spaces of rank one, Illinois J. Math. 21 (1977), 1-41.

10. C. Gérard, Asymptotique des poles de la matrice de scattering pour deux obstacles strictement convexes, Université de Paris-Sud, preprint, 1989.

11. D. Hejhal, The Selberg trace formula for $\operatorname{PSL}(2, \mathbf{R})$. I, Lecture Notes in Math., vol. 548, Springer-Verlag, Berlin, Heidelberg, New York, 1976.

12. M. Ikawa, On the poles of the scattering matrix for two strictly convex obstacles, J. Math. Kyoto Univ. 23 (1983), 127-194.

13. P. Lax and R. S. Phillips, The asymptotic distribution of lattice points in Euclidean and non-Euclidean spaces, J. Funct. Anal. 46 (1982), 280-350.

14. __ Translation representation for automorphic solutions of the non-Euclidean wave equation. I, II, III, Comm. Pure. Appl. Math. 37 (1984), 303328; 779-813 and 38 (1985), 179-208.

15. __ Translation representation for automorphic solutions of the non-Euclidean wave equation. IV, Stanford University, preprint, 1990.

16. N. Mandouvalos, The theory of Eisenstein series for Kleinian groups, The Selberg Trace Formula and Related Topics (D. Hejhal, P. Sarnak, and A. Terras, eds.), Contemp. Math., vol. 53, Amer. Math. Soc., Providence, RI, 1986, pp. 357-370.

17. __, Spectral theory and Eisenstein series for Klenian groups, Proc. London Math. Soc. 57 (1988), 209-238.

18. __ Scattering operator, Eisenstein series, inner product formula, and Maass-Selberg relations for Kleinian groups, Mem. Amer. Math. Soc. 400 (1989), 1-87.

19. __ Scattering operator and Eisenstein integral for Kleinian groups, Math. Proc. Cambridge Philos. Soc. (1990)

20. H. P. McKean, Selberg's trace formula as applied to a compact Riemann surface, Comm. Pure. Appl. Math. 25 (1972), 225-246.

21. R. B. Melrose, Polynomial bounds on the number of scattering poles, J. Funct. Anal. 53 (1983), 287-303.

22. __ Polynomial bounds on the distribution of poles in scattering by an obstacle, Journées "Équations aux derivées partielles," Saint-Jean-de-Monts, 1984.

23. R. Mazzeo and R. Melrose, Meromorphic extension of the resolvent on complete spaces with asymptotically constant negative curvature, J. Funct. Anal. 75 (1987), 260-310

24. S. J. Patterson, The Laplacian operator on a Riemann surface. I, II, III, Compositio Math. 31 (1975), 83-107; 32 (1976) 71-112; and 33 (1976), 227-259. 
25. __ The Selberg zeta-function of a Kleinian group, Number Theory, Trace Formulas, and Discrete Groups: Symposium in honor of Atle Selberg, Oslo, Norway, July 14-21, 1987, New York, Academic Press, 1989, pp. 409-442.

26. P. A. Perry, The Laplace operator on a hyperbolic manifold. II. Eisenstein series and the scattering matrix, J. Reine Angew. Math. 398 (1989), 67-91.

27. _ The Selberg zeta function and a local trace formula for Kleinian groups, J. Reine Angew. Math. 410 (1990), 116-152.

28. _ Trace formulas and scattering poles for the Laplacian on a hyperbolic manifold. To appear in the Proceedings of an International Conference on "Spectral and scattering theory of differential operators" held in honor of Shmuel Agmon at Hebrew University, Jerusalem, June 12-15, 1990.

29. _ On the distribution of scattering poles for Kleinian groups (in preparation).

30. A. Selberg, Harmonic analysis and discontinuous groups in weakly symmetric Riemannian spaces with applications to Dirichlet series, J. Indian Math. Soc. 20 (1956), 47-87.

31. D. Ruelle, Zeta-functions for expanding maps and Anosov flows, Invent. Math. 34 (1976), 231-242.

32. S. Smale, Differentiable dynamical systems, Bull. Amer. Math. Soc. (N.S.) 73 (1967), 747-817.

33. M. Zworski, Distribution of poles for scattering on the real line, J. Funct. Anal. 73 (1987), 277-296.

34. _ Sharp polynomial bounds on the number of scattering poles of radial potentials, MIT, preprint, 1989.

35. __ Sharp polynomial bounds on the number of scattering poles, MIT, preprint, 1989.

Department of MAthematics, University of KentUCKy, Lexington, KeNTUCKY 40506-0027 
